\title{
Automated live cell imaging of cell migration across a microfluidic-controlled chemoattractant gradient
}

\author{
The quantification of cell-migration behavior in response to gradients is critical for understanding \\ cellular movement and development. The CellASIC ${ }^{\circledR}$ ONIX microfluidic platform offers a microfluidic \\ gradient plate to enable precision-controlled chemoattractant diffusion across perfusion barriers, \\ enabling a quantitatively defined diffusion gradient stable enough for long-term, live cell analysis. \\ Using the system, we studied the effect of a serum gradient on metastatic breast cancer cell migration \\ distance, velocity and degree of chemotaxis.
}

\section{A novel platform for quantifying chemotaxis}

Cell migration is stimulated and directed by the interaction of cells with extracellular matrix, neighboring cells or chemoattractants and is key to processes such as development and metastasis. Mechanisms of migration can be best understood by visualizing and quantifying cellular responses to modulators of migration.

The most widely accepted cell-migration assay is the membranebased transwell assay. However, very steep gradients can form along a single axis perpendicular to the surface of the membrane, resulting in a lower-than-expected difference in chemoattractant concentration between upper and lower wells. As a result, this method is unsuitable for correlating specific cell responses with particular gradient characteristics (slope, concentration, temporal evolution, etc.). Furthermore, gradients are not very stable under 'static' cell-culture conditions, precluding live cell imaging. We created a quantitatively defined, stable diffusion gradient for long-term, live cell imaging using a novel microfluidic gradient plate. This plate, designed for us on the CellASIC ${ }^{\circledR}$ ONIX microfluidic platform, enables precision-controlled chemoattractant diffusion across perfusion barriers to create a spatial gradient in each culture area (Fig. 1). For each culture area, perfusion inlets and outlets form a continuous-flow 'infinite' source and sink that maintains a stable concentration-gradient profile for days. The flexible format of the plate makes it possible to change the gradient directionality, turn gradients on and off, and toggle between gradient and single-solution exposure.

Using the power of long-term, live imaging of cells on a stable chemoattractant gradient, we studied the effect of a serum gradient on metastatic breast cancer cell migration distance, velocity and degree of chemotaxis.

Philip Lee, Cindy S Y Chen, Terry Gaige \& Paul J Hung

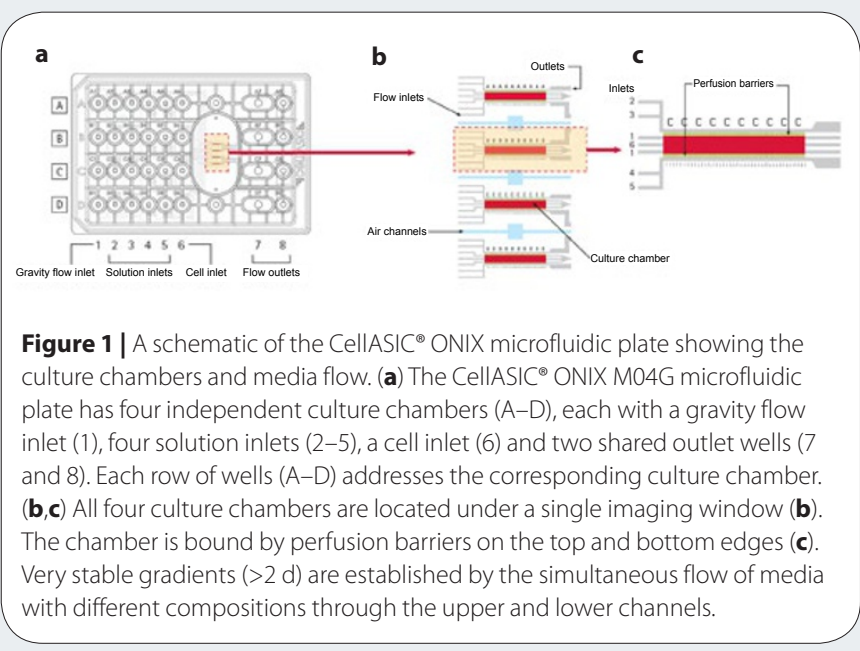

Perfusion cell culture, chemotaxis assays and live cell imaging

Trypsinized MDA-MB-231 cells were introduced into the four preprimed chambers of the microfluidic plate using pressure-driven cell loading. Plates were placed in a standard incubator for $1 \mathrm{~h}$ to allow cell attachment. The loaded plate was then sealed to the CelIASIC ${ }^{\circledR}$ ONIX F84 manifold and placed on the stage of the microscope. The manifold connected the plate to the CellASIC ${ }^{\circledR}$ ONIX microincubator controller. The entire system was controlled using CellASIC ${ }^{\circledR}$ ONIX FG software.

For automated media perfusion, each experiment involved three phases: complete medium feeding, serum starvation and exposure to a $0-10 \%$ fetal bovine serum (FBS) gradient. The different media were loaded into various inlet wells, and flow rates, pressures and times were preprogrammed using CellASIC ${ }^{\circledR}$ ONIX FG software. 
All images and videos were acquired at $10 \times$ magnification. Timelapse images were captured at 20-min intervals. Images were analyzed using ImageJ software (US National Institutes of Health (NIH)) in combination with Manual Tracking (NIH) and Chemotaxis Tool (ibidi) plug-ins. In each case, 50 representative cells were tracked for migratory properties across a sequential series of 36 images (12 h in culture).

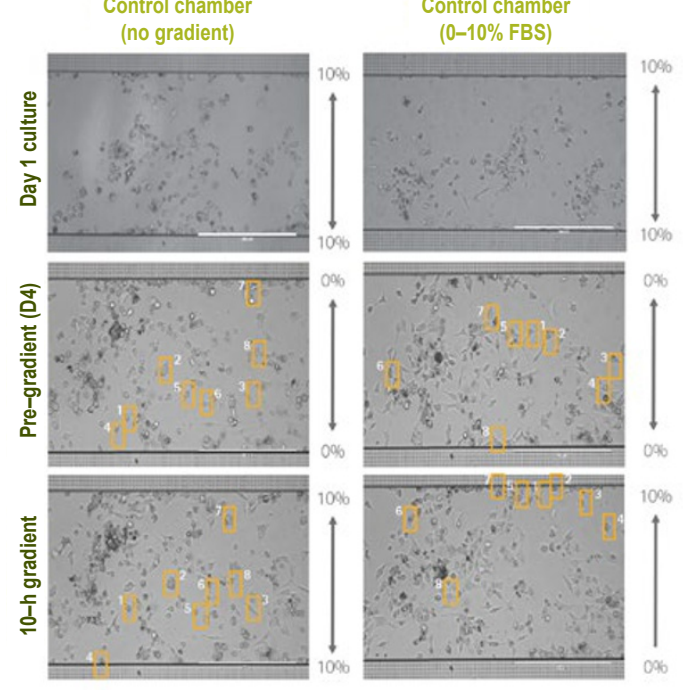

Figure 2 |MDA-MB-231 cell migration after exposure to a stable FBS gradient. Cells were incubated in complete medium (control chamber) or exposed to a $0-10 \%$ FBS gradient (gradient chamber) established along the $y$-axis. Cells post-loading are shown at the top ( $3 \mathrm{~d}$ in complete medium followed by $24 \mathrm{~h}$ of serum starvation (no FBS)). Images in the bottom row were taken $12 \mathrm{~h}$ after gradient induction

\section{Measurement of cell migration}

To demonstrate the value of our platform, we monitored the movement of individual MDA-MB-231 metastatic human breast cancer cells in response to spatial changes in FBS concentration (Fig. 2). In general, in cultures exposed to an FBS gradient, cells moved toward the upper barrier of the cell chamber. By contrast, control cells cultured in the absence of a spatial serum gradient demonstrated less movement overall and moved in far more random directions. The boxes (numbered 1-8) were used to identify particular cells and demonstrate their overall movement over $12 \mathrm{~h}$. Cells exposed to FBS gradients tended to move preferentially toward sources of higher FBS concentration.

Using ImageJ software, we tracked the migratory properties of individual cells. For each culture condition, 50 cells were monitored for $12 \mathrm{~h}$ (Fig. 3). Cells moved far less in stable medium than when exposed to a nutrient gradient, and cell migration was significantly more directed when a gradient was established in the chamber. When we analyzed cell migration distance and directionality, we found that cells exposed to an FBS gradient migrated more than threefold farther from their site of origin than did cells in constant medium, suggesting that the cells were actively seeking the nutrient-rich medium at the high-FBS-concentration end of the gradient.
In general, cells migrated with a slightly higher velocity in chambers where an FBS gradient was established (calculated from Fig. 3). Interestingly, we observed that (1) cells tended to migrate farther in cultures of lower cell density, and (2) members of a cell cluster migrated faster than their isolated counterparts. This latter response might have been caused by localized cell-cell communication in the microenvironment, as previously reported in studies analyzing the relationship between cell-cell interactions and migration rates ${ }^{1-3}$. a

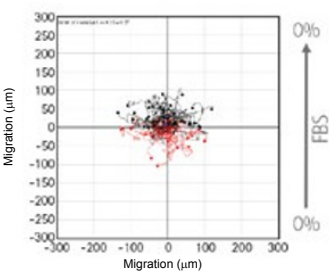

C

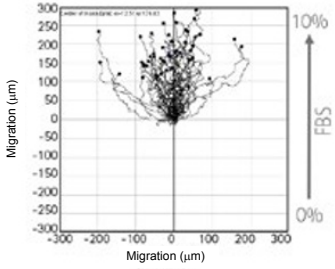

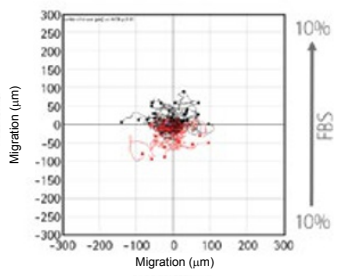

d

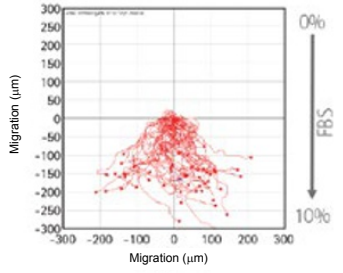

Figure 3 | Plots of migration in $x$ - and $y$-directions highlighting the impact of the FBS gradient on MDA-MB-231 cell movement. (a-d) Plots of migration observed with (a) 0\% FBS (no gradient), (b) 10\% FBS (no gradient), (c) 10-0\% FBS (bottom-to-top gradient) and (d) 0-10\% FBS (top-to-bottom gradient). In each plot, black and red lines denote cells with a net upward or downward movement relative to the $y$-axis, respectively.

\section{Conclusions}

Microfluidic control of cell culture conditions and chemoattractant gradients, while maintaining an uninterrupted optical path to cells, enables precise, dynamic quantitation of cell migration in response to stimuli. Our approach may enable future studies comparing the effects of signaling molecules and growth factors on the migration propensities of cells in tumors, wounds, developing tissues, immune responses and other biological systems defined by active cell migration.

\footnotetext{
1. Han, L. et al. Biomaterials 34, 975-984 (2013).

2. Shih, W. and Yamada, S. Cell Adh. Migr. 6, 513-517 (2012).

3. Shih, W. and Yamada, S. J. Cell Sci. 125, 3661-3670 (2012)
}

This article was submitted to Nature Methods by a commercial organization and has not been peer reviewed. Nature Methods takes no responsibility for the accuracy or otherwise of the information provided. 